Gefässchirurgie 2021 - 26:644-646 https://doi.org/10.1007/s00772-021-00846-1 Angenommen: 1. November 2021 Online publiziert: 23. November 2021 (c) The Author(s), under exclusive licence to Springer Medizin Verlag $\mathrm{GmbH}$, ein Teil von Springer Nature 2021

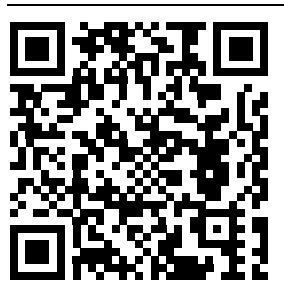

QR-Code scannen \& Beitrag online lesen

\section{Rauchentwöhnung und Harm Reduction, was ist gesichert?}

\author{
Veranstaltung im Rahmen der 37. Jahrestagung der \\ Deutschen Gesellschaft für Gefäßchirurgie und \\ Gefäßmedizin am 15.10.2021 im Kongresszentrum \\ Mannheim
}

Martin Storck

Klinik für Gefäß- und Thoraxchirurgie, Städtisches Klinikum Karlsruhe, Karlsruhe, Deutschland
Die wissenschaftliche Veranstaltung fand im Rahmen des 37. Jahreskongresses der Deutschen Gesellschaft für Gefäßchirurgie und Gefäßmedizin in Mannheim im Kongresszentrum statt. Die Organisation und Sitzungsleitung führte Herr Prof. Dr. Martin Storck, Direktor der Klinik für Gefäßund Thoraxchirurgie im Klinikum Karlsruhe durch. Co-Moderator war Herr Dr. Bernd Werse, Fachbereich Erziehungswissenschaften am Institut für Sozialpädagogik und Erwachsenenbildung an der Universität Frankfurt.

Weitere hochranginge nationale und internationale Referent*innen auf dem Gebiet der Rauchentwöhnung und Public Health waren eingeladen: Dr. Elke Pieper, wissenschaftliche Mitarbeiterin am Bundesinstitut für Risikobewertung, Frau Prof. Dr. Ute Mons, Professur für kardiovaskuläre Epidemiologie des Alterns, Universität Köln, und Frau Dr. Leonie Brose, Nationales Suchtzentrum, Institut für Psychiatrie, Kings College London, sowie Herr Dr. Thomas Hering, Facharzt für Lungenheilkunde, Beauftragter für Tabakrisiken-Fragen und Tabakentwöhnung im Bundesverband der Pneumologen und Mitglied der Arbeitsgruppe Tabakprävention der Deutschen Gesellschaft für Pneumologie.

Rauchen ist einer der Hauptrisikofaktoren für Arteriosklerose und kardiovaskuläre Erkrankungen. Trotz dieses Wissens gelingt es vielen ärztlichen Behandlern nicht, $\mathrm{Pa}$ tienten das Rauchen abzugewöhnen, obwohl dies eine effektive und empfohlene Präventionsmaßnahme darstellt. Auch in der Rehabilitationsmedizin sind die Er- folgsraten der Rauchentwöhnung gering. Wo die Rauchentwöhnung nicht gelingt, kann das Prinzip der "Harm Reduction“ ein alternativer Weg sein, was bedeutet, dass auf eine deutlich geringer schädliche Alternative ohne Tabakverbrennung ausgewichen wird, entweder auf eine medikamentöse Nikotinersatztherapie oder auf E-Zigaretten und Tabakerhitzer. In dem Symposium wurden die Prinzipien der Harm Reduction und auch die Rolle und Bewertung der E-Zigaretten und Tabakerhitzer ausführlich diskutiert.

Nach einem Einführungsvortrag durch Prof. Storck, in welchem die Unterschiede zwischen der Gefährlichkeit von Nikotin (Suchtstoff ohne Krebspotenzial) und Tabakrauch (mit Risiko Arteriosklerose und Krebserkrankung) dargelegt wurden, folgte zunächst ein Vortrag von Frau Dr. Elke Pieper vom Bundesinstitut für Risikobewertung. In diesem Vortrag wurde wissenschaftlich klargestellt, dass der Gehalt an Schadstoffen in der Emission von E-Zigaretten und Tabakerhitzern um mehr als 85 bis $95 \%$ geringer ist als bei Zigarettenrauch, was sich auf die gesundheitlichen Risiken auswirken muss. Konkrete klinische Korrelationen zu diesen toxikologischen Daten liegen nicht vor, insbesondere noch keine Langzeitdaten. Nach Aussage von Frau Dr. Pieper ist davon auszugehen, dass bei bestimmungsgemäßem Gebrauch aller Arten von E-Zigaretten und Tabakerhitzern die gesundheitlichen Risiken deutlich geringer sind als beim Tabakrauchen. Zurzeit gibt es keine Daten, die besagen, dass von den in der EU verwendeten Aroma- 
substanzen für E-Liquids zusätzliche gesundheitliche Risiken ausgehen.

Ein weiterer Beitrag von Dr. Hering aus Berlin widmete sich dem Thema, ob E-Zigaretten und Tabakerhitzer zur Rauchentwöhnung wirklich geeignet sind. Herr Dr. Hering wies in seinem Vortrag insbesondere darauf hin, dass Patienten mit schwerer und End-stage-COPD(Chronisch obstruktive Lungenerkrankung) sich durch Weiterrauchen "zu Tode rauchen", das heißt, dass gerade bei den Patienten mit schlechter Lungenfunktion ein rechtzeitiger Wechsel auf schadstoffärmere Ersatzprodukte, insbesondere E-Zigaretten, sinnvoll ist. Damit steht er in gewissem Gegensatz zu den Aussagen von Fachgesellschaften, die jede Art von Inhalation und E-Zigaretten grundsätzlich ablehnen, jedoch in Übereinstimmung zum Beispiel mit den Positionen des Bundesinstitutes für Risikobewertung (BfR), der Gesundheitsbehörde in Großbritannien sowie der wissenschaftlichen Fachgesellschaft zu Tabak- und Nikotin-Fragen (SRNT). Die generelle Ablehnung der E-Zigarette (auch kürzlich von der WHO formuliert) wurde von allen Referenten dieses Symposiums kritisch hinterfragt.

Frau Prof. Mons aus Köln berichtete in ihrem ausführlichen Referat wie die deutsche Tabakkontrolle im europäischen Vergleich dasteht. Spitzenreiter, was Tabakkontrolle und konkrete Rauchentwöhnungsangebote angeht, ist das Vereinigte Königreich. Gemäß einer europaweiten Tabakkontrollskala, die 41 Länder vergleicht, liegt Deutschland hingegen auf dem letzten Platz. Entsprechend sinken die Raucherquoten in Deutschland nur leicht und insbesondere in der Bevölkerung des mittleren und höheren Alters sind im Zeitverlauf sogar steigende Raucherquoten zu beobachten. Frau Prof. Mons sieht hier entsprechend einen immensen Handlungsbedarf für die Gesundheitspolitik, um insbesondere diese bislang vernachlässigten Altersgruppen zur Rauchentwöhnung zu motivieren. Harm-Reduction-Strategien können Tabakkontrollstrategien hierbei sinnvoll ergänzen.

Frau Dr. Leonie Brose aus London wies auf die Schwierigkeiten der selbst versuchten Rauchentwöhnung hin. So zeigte eine Erhebung, dass von 100 Rauchern ohne Coaching maximal 67 Patienten willens sind aufzuhören, 34 jedoch nur einen ernsthaften Versuch unternehmen und nur ein einziger Raucher es tatsächlich schafft, langfristig mit dem Rauchen aufzuhören. Diese Erfolgsraten sind so gering, dass hier unbedingt regulatorische und gesundheitspolitische Maßnahmen ergriffen werden müssen. Insbesondere wies sie auf die Tatsache hin, dass das Nikotin als Suchtstoff in den Ersatzprodukten ausreichend zur Verfügung gestellt werden muss.

Zuletzt wies Dr. Bernd Werse aus Frankfurt darauf hin, dass die sogenannte Gateway-Theorie - zu Deutsch etwa: Einstiegsdrogentheorie - bei Jugendlichen in Bezug auf E-Zigaretten als Einstieg in das Zigarettenrauchen nicht bestätigt werden kann. Insgesamt hat den Ergebnissen diverser repräsentativer Befragungen zufolge - die Zahl der jugendlichen Raucher in Deutschland gerade nach Einführung derE-Zigaretten nochmals so stark abgenommen, dass allein deshalb nicht von einem relevanten Einstiegseffekt geredet werden kann. Mit den langfristigen Daten einer Repräsentativerhebung in Frankfurter Schulen konnte zudem gezeigt werden, dass Jugendliche, die E-Zigaretten vor dem Rauchen ausprobieren, vergleichsweise selten abhängige Raucher werden. Herr Dr. Werse begrüßte ein Werbeverbot für jede Art von suchtmittelartigen Produkten, wies aber darauf hin, dass im Prinzip dann auch ein generelles Werbeverbot für Alkohol und andere psychoaktive Substanzen gefordert werden müsste.

Prof. Storck wies abschließend darauf hin, dass in Zukunft durch Einflussnahme von Fachgesellschaften und auch durch politische Einflussnahmen Fortschritte auf diesem Gebiet erzielt werden müssen, da sonst die gesundheitlichen Schäden durch das Rauchen fortbestehen und zunehmen werden und die deutschen Raucher und die Gesundheitspolitik in Deutschland weiter Schlusslichter in Europa bleiben.

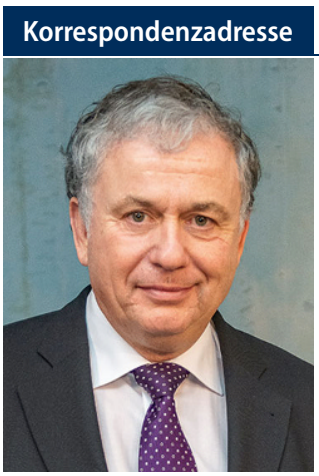

Prof. Dr. Martin Storck

Klinik für Gefäß- und Thoraxchirurgie, Städtisches Klinikum Karlsruhe

Moltkestraße 90, 76133 Karlsruhe, Deutschland martin.storck@klinikum-karlsruhe.de

Interessenkonflikt. M. Storck gibt an, dass kein Interessenkonflikt besteht. 


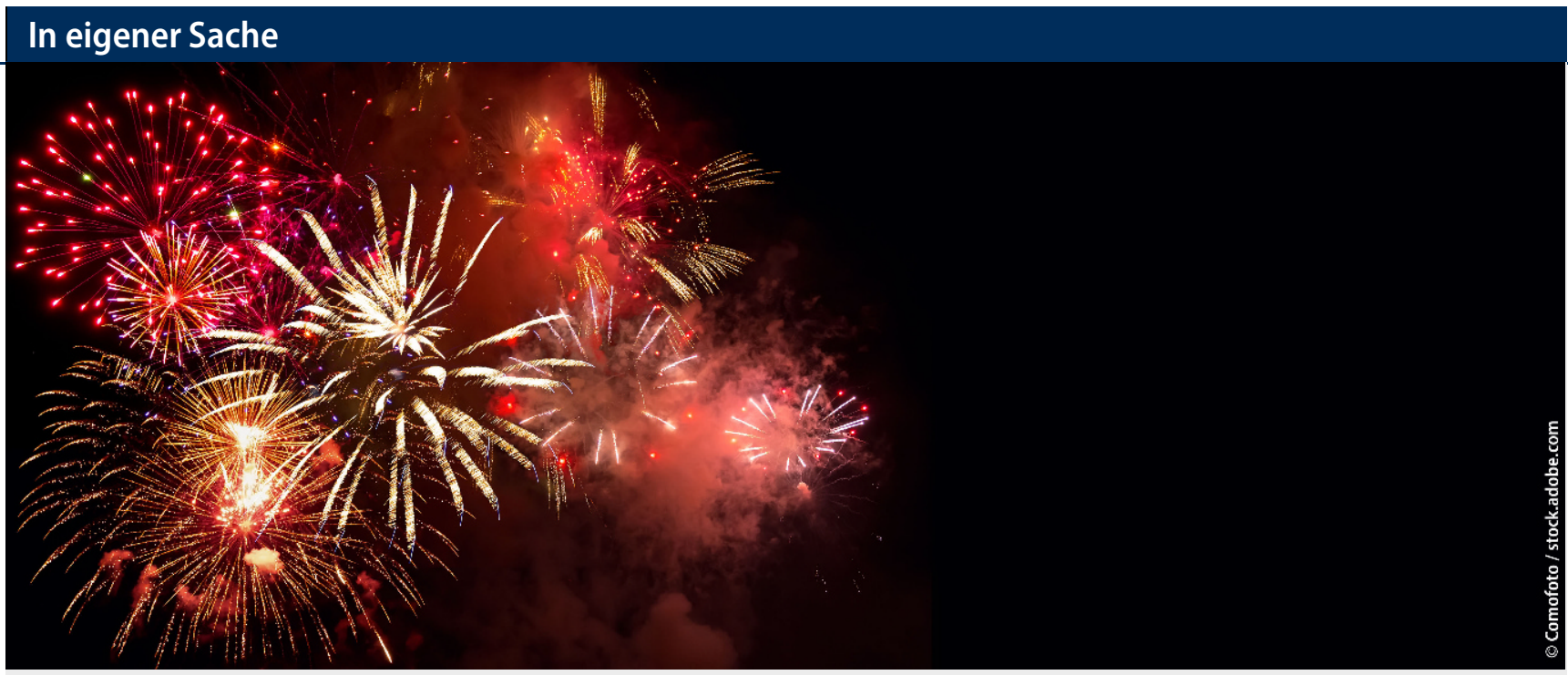

\section{Julius-Springer-Preis für Gefäßmedizin 2021}

\section{Beste Originalarbeit aus Gefässchirurgie mit Julius-Springer-Preis für Gefäßmedizin ausgezeichnet: Der Beitrag „Roboterbasierte Ultraschallsteuerung" von Herrn PD Dr. Mark Preuß gewinnt den Preis für 2021}

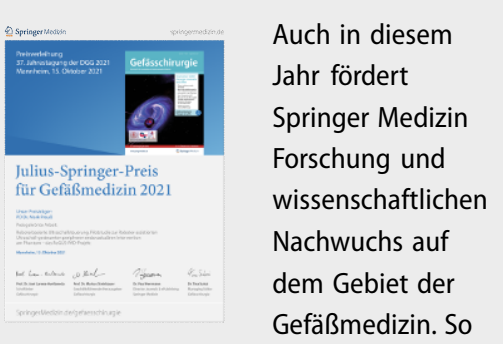

vergibt die Fachverlagsgruppe bereits zum

12. Mal in Folge den Julius-Springer-Preis für Gefäßmedizin. Diesjähriger Preisträger ist der Mediziner PD Dr. Mark Preuß für seine Orginalarbeit: „Roboterbasierte Ultraschallsteuerung. Pilotstudie zur Roboterassistierten Ultraschall-gesteuerten peripheren endovaskulären Intervention am Phantom - das RoGUS-PAD-Projekt". Erstmalig hat sich ein Experte mit der Möglichkeit eines robotischen Ultraschallassistenzsystems bei peripheren endovaskulären Interventionen auseinandersetzt. Für seinen herausragenden Beitrag, erschienen in Gefässchirurgie 05/2020, erhält Preuß ein Preisgeld von Euro 1.000. Die Preisverleihung fand am 15. Oktober während der 37. Jahrestagung der Deutschen Gesellschaft für Gefäßchirurgie und Gefäßmedizin in Mannheim statt.

Motivation zum sogenannten RoGUS-PADProjekt, Robotic Guided Ultrasound System für Peripheral Arterial Disease, war die Ausgangsituation, dass bei peripheren arteriellen Verschlusskrankheiten in herkömmlichen Untersuchungen Röntgenkontrastmittel und
Röntgenstrahlungen zum Einsatz kommen. Die zu hohe Strahlenbelastung geht zu Lasten des Untersuchten und der Untersuchenden. Das Röntgenkontrastmittel kann zu einer Schädigung der Nieren des Patienten führen. Im Fokus der Preisarbeit standen alternative Navigationsmethoden, die im Sinne einer Weiterentwicklung in der Gefäßmedizin von hoher Relevanz sind.

In seiner ausführlichen Studie verfolgte Preuß die Idee, ein roboterbasiertes Assistenzsystem zu prüfen. Da ein manuell geführter Schallkopf wenig praktikabel ist, sollte ein automatisierter Schallkopf zur klinischen Routine weiterentwickelt werden. Der Einsatz eines semiautomatischen 2-D-Ultraschallscan zeigte am Phantom bei 27 von 30 Scans erfolgreiche Ergebnisse.

Optimistisch blicken die Experten hier in die Zukunft: Laut ihrer Einschätzung könnte nun unter Nutzung von Technologien wie "Deep-learning-Algorithmen" eine baldige vollautomatische Nutzung eines Schallkopfes bei gefäßmedizinischen Untersuchungen zur Verfügung stehen.

Jedes Jahr fördert die Fachverlagsgruppe Springer Medizin Nachwuchswissenschaftler in den verschiedenen Fachgebieten. Der Julius-Springer-Preis für Gefäßmedizin wird von der Fachverlagsgruppe zusammen mit der wissenschaftlichen Leitung der
Fachzeitschrift Gefässchirurgie vergeben für eine herausragende Arbeit, die dem Fachgebiet und seiner Forschung dienlich ist.

Den Beitrag "Roboterbasierte Ultraschallsteuerung", erschienen in Gefässchirurgie (5/2020) 25:345-351, können Sie hier herunterladen:

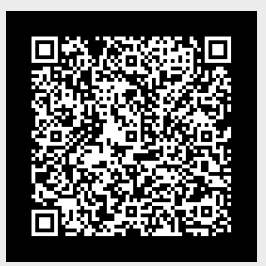

